\title{
Comparison of the Enamel Surface Roughness from Different Polishing Methods: Scanning Electron Microscopy and Atomic Force Microscopy Investigation
}

\author{
Kiatanan Sugsompian ${ }^{1}$ Ratchawan Tansalarak ${ }^{2}$ Thosapol Piyapattamin ${ }^{2}$ \\ ${ }^{1}$ Kosum Phisai Hospital, Amphoe Kosum Phisai, Maha Sarakham, \\ Address for correspondence Thosapol Piyapattamin, DDS, PhD, \\ Thailand \\ 2Department of Preventive Dentistry, Faculty of Dentistry, \\ Naresuan University, Phitsanulok, Thailand \\ Dip., Thai Board of Orthodontics, Department of Preventive Dentistry, \\ Faculty of Dentistry, Naresuan University, Phitsanulok 65000, Thailand \\ (e-mail: thosapolp@nu.ac.th).
}

Eur J Dent 2020;14:299-305

\begin{abstract}
Keywords

- enamel surface roughness

- debonding

- polishing
\end{abstract}

Objective This study aimed to compare the enamel surface roughness created by four polishing methods after debonding, by using scanning electron microscopy (SEM) and atomic force microscopy (AFM).

Materials and Methods Four experimental polishing groups (Sof-Lex disc, SD; sandblaster, SB; tungsten carbide bur, TB; and white stone bur, WB) and one control group were selected from 100 premolars ( $n=20 /$ group). The experimental teeth were bonded with a bracket, thermocycled, and debonded. Residual adhesive was removed by either of the respective methods. Pre and postdebonding root mean square (Rq) values were obtained from AFM evaluations. All specimens were examined and evaluated with SEM using a modified enamel surface index (modified ESI).

Statistical Analysis Differences among the polishing methods were compared with analysis of variance and Fisher's least significant difference test at $p<0.05$.

Results Both microscopic evaluations indicated that the surface with the greatest roughness herein belonged to the SD group, followed by that for SB, TB, and WB groups. AFM measurements indicated a maximum postdebonding $\mathrm{Rq}$ herein for the WB group and a significantly greater surface roughness for the TB and WB groups than for the SD and SB groups. Among the experimental groups, SEM followed by modified ESI evaluations revealed similar data to those obtained with AFM. Significant differences were seen among all paired groups, except for that between the SB and TB groups.

Conclusion Within the limitations of this study, all four polishing methods were concluded to be clinically acceptable for removing residual orthodontic adhesives.

\section{Introduction}

After a fixed orthodontic treatment is completed, a debonding procedure, which includes removing the appliances and cleaning all residual adhesive from enamel surfaces, must be performed by an orthodontist ${ }^{1}$ and is dependent on the preference of the operator. ${ }^{2}$ Several methods for the procedure and their associated devastation of the enamel were developed with various indices and documented. ${ }^{3}$

Utilization of a hand scaler, an ultrasonic scaler, a laser, or a sandblaster, as well as some rotary instruments with a green stone bur, a white stone bur, a diamond bur, a tungsten carbide bur, a composite bur, or a Sof-Lex disc (3M ESPE; Minnesota, United States) was suggested for removing 
residual adhesive. ${ }^{4-10}$ Because of its cost effectiveness, a white stone bur was recommended in a report. ${ }^{11} \mathrm{~A}$ tungsten carbide bur, particularly that with 12 or 20 flutes, is reported as the gold standard for debonding because of its creation of a satisfactory finished surface. ${ }^{12,13}$ Flexible and very thin Sof-Lex disc contains coarse, medium, fine, and superfine refinement degrees. Compared with those by other dental burs, a Sof-Lex disc produces a relatively smooth postpolished surface. ${ }^{5,14}$ A sandblaster with aluminum oxide particles neatly removes stains on the enamel surface,,$^{15}$ increases orthodontic bond strength, ${ }^{16}$ and can be used for debonding with favorable outcomes. ${ }^{9,17}$

While some indices are used for quantitative assessments of residual adhesive on enamel surfaces, ${ }^{18,19}$ the composite remnant index (CRI) is regarded as an uncomplicated one with high reliability ( - Table $\mathbf{1}) .{ }^{12}$ Enamel surface roughness can be visualized by profilometry, ${ }^{9}$ rugosimetry, ${ }^{14}$ scanning electron microscopy, ${ }^{10}$ and atomic force microscopy. ${ }^{20}$ Profilometry, rugosimetry, and atomic force microscopy provide three-dimensional (3D) numerical data of the surface roughness for subsequent evaluation. In contrast, scanning electron microscopy gives two-dimensional (2D) information, and thus visual enamel evaluation indices are required to conduct statistical analysis. The surface roughness index ${ }^{12}$ and the enamel damage index ${ }^{21}$ depend on only the enamel roughness and damage, respectively. The enamel surface rating system contains so many scores that a researcher may get confused during evaluation..$^{22}$ Based on the presence of perikymata, the enamel surface index (ESI) is likely to cause a bias during assessing the enamel surface on a young tooth. ${ }^{23}$ With its simple point scores and demonstrable reproducibility, the modified enamel surface index (modified ESI) allows a researcher to easily recognize the appropriate value and use it for subsequent interpretations (- Table 2). ${ }^{24}$

Despite it being the hardest tissue in the human body, ${ }^{25}$ enamel can be destroyed by some dental treatment

Table 1 Scoring system and interpretations of composite remnant index ${ }^{12}$

\begin{tabular}{|l|l|}
\hline Score & Interpretation \\
\hline 0 & No composite left \\
\hline 1 & $1 / 4$ (or less) of composite left \\
\hline 2 & $1 / 2$ (or less) of composite left \\
\hline 3 & $3 / 4$ (or less) of composite left \\
\hline 4 & All of the composite remained \\
\hline
\end{tabular}

Table 2 Scoring system and interpretations of modified enamel surface index ${ }^{24}$

\begin{tabular}{|l|l|}
\hline Score & Interpretation \\
\hline 0 & Perfect surface (no scratches) \\
\hline 1 & Satisfactory surface (fine scratches) \\
\hline 2 & $\begin{array}{l}\text { Acceptable surface (several marked areas and some } \\
\text { deeper scratches) }\end{array}$ \\
\hline 3 & $\begin{array}{l}\text { Imperfect surface (several distinct deep and coarse } \\
\text { scratches, and/or composite remaining) }\end{array}$ \\
\hline
\end{tabular}

procedures, particularly by removal of orthodontic adhesive. ${ }^{3}$ Although the recovery of a postpolished surface to its prebonded morphology is hardly achieved, the iatrogenic enamel damage needs to be minimized. Consequently, explorations of methods that cause the least harm, if any, to the enamel surface are needed. Hence, this in vitro study aims to compare and evaluate the enamel surface roughness from four different polishing methods after bracket detachment by using scanning electron microscopy and atomic force microscopy.

\section{Materials and Methods}

The study was approved by the Naresuan University Ethical Committee; Naresuan University, Phitsanulok, Thailand.

\section{Specimen Preparation}

This comparative in vitro study used 100 human maxillary first premolars extracted to serve an orthodontic purpose. The inclusion criteria for the crowns included having a normal morphology, flawless enamel, and no history of orthodontic treatment. Those with buccal surfaces that possessed fluorosis, a crack line, a fracture, a carious lesion, a restorative material, an abfraction, or an abrasion were excluded. After cleansing with distilled water, the teeth were stored in $0.1 \%$ thymol solution until use. Their roots up to the cervices were sectioned by using a cutting machine coupled with distilled water irrigation. Each remaining crown was horizontally embedded in a plastic pipe, with an exposure of its buccal surface 1.0-1.5 mm above the rims of the pipe. The crown was then fixed by using self-curing acrylic resin (Orthocryl; Dentaurum, Ispringen, Germany) as shown in - Fig. 1. After cleansing their buccal surfaces with a slurry of pumice, all specimens were randomly divided into five groups ( $n=20 /$ group) comprising one control and four experimental groups (Sof-Lex disc; sandblaster [Parkell, New York, United States]; tungsten carbide bur [Reliance Orthodontic Products; Illinois, United States]; and white stone bur [Shofu; Kyoto, Japan]). Their details are provided in - Table 3.

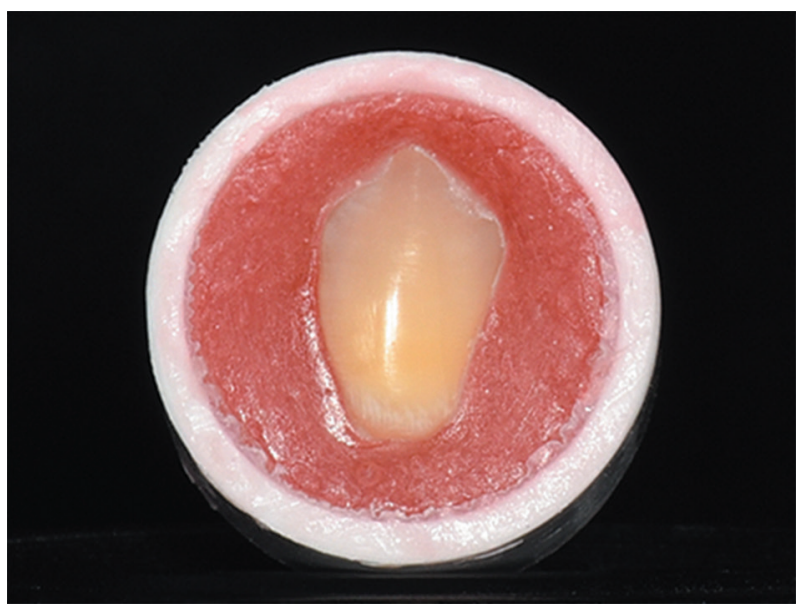

Fig. 1 Human maxillary first premolar crown embedded in a plastic pipe and fixed by self-curing acrylic resin with its buccal surface 1.0 to $1.5 \mathrm{~mm}$ above the pipe rim. 
Table 3 Details of each group

\begin{tabular}{|l|l|l|l|}
\hline Group & Material for polishing & Handpiece speed (rpm) & Coolant type \\
\hline Control & NA & NA & NA \\
\hline Sof-Lex disc & Sof-Lex disc & Low $(30,000)$ & Air \\
\hline Sandblaster & $50-\mu m$ aluminum oxide & NA & NA \\
\hline Tungsten carbide bur & Tungsten carbide bur & High $(120,000)$ & Water \\
\hline White stone bur & Dura-white stone bur & High $(120,000)$ & Water \\
\hline
\end{tabular}

Abbreviations: NA, not applicable; rpm, revolutions per minute; $\mu \mathrm{m}$, micron.

\section{Bonding, Thermocycling, and Debonding}

For each of the 80 experimental teeth, the buccal surface enamel center was etched with $35 \%$ phosphoric acid gel (Scotchbond Etchant; 3M ESPE, Minnesota, United States) for 15 seconds, rinsed with a constant water spray for 10 seconds, and dried with oil-free compressed air jets for 10 seconds. Using a disposable microbrush (Microbrush International; Wisconsin, United States), a thin and uniform film of primer/adhesive (Transbond XT Primer-Adhesive; 3M Unitek, Californian, United States) was applied to the etched enamel. A sufficient amount of adhesive (Transbond Plus Color Change Adhesive; 3M Unitek) was placed on the meshes of a metallic bracket (3M Unitek), and the bracket was then positioned at the center of the long axis of the crown. To produce a similar adhesive thickness on each tooth, the bracket was compressed with a constant light force, and the excess amounts were gently removed by a dental explorer. After adhesive polymerization by using a light-curing unit (SPEC3; Coltene, Ohio, United States), all specimens were stored in distilled water for 24 hours at $37^{\circ} \mathrm{C}$.

The control and experimental teeth were subjected to 500 thermal cycles from $5-50^{\circ} \mathrm{C}$ for 20 seconds for each bath and at $25^{\circ} \mathrm{C}$ for $5-10$ seconds for interbath movement to simulate an intraoral environment, ${ }^{26}$ according to the International Standard Organization standard.

The subsequent debonding and polishing procedures for the 80 experimental teeth were performed. After debonding all brackets by using a conventional bracketremover (678-219; Hu-Friedy, Illinois, United States) with a peeling force, the residual adhesives were assessed under a stereomicroscope (Olympus SZH10; Olympus, Tokyo, Japan) at a $25 \times$ magnification and evaluated by determining the CRI. Each experimental tooth surface was polished by either of the four methods (-Table 3 ). For the Sof-Lex disc, tungsten carbide bur, and white stone bur groups, the residuals were polished by pressing the shank of the burs parallel to each tooth surface and moving the handpiece in a mesial-to-distal direction. For the sandblaster group, a sandblaster was used with its tip perpendicular to and $10 \mathrm{~mm}$ over the tooth surface with an air pressure of $\sim 7 \mathrm{~kg} / \mathrm{cm}^{2}$. Using the oil-free compressed air jets, the specimens were rinsed by distilled water and air-dried. After careful inspection by the naked eye under the light of the curing unit, a complete adhesive removal from the tooth surface was reconfirmed under the stereomicroscope at a $25 \times$ magnification.

\section{Atomic Force Microscopy}

To determine the predebonding root mean square roughness $(\mathrm{Rq})$, the teeth undergoing cleansing with a pumice slurry were investigated by using an atomic force microscope (Flex-Axiom; Nanosurf, Liestal, Switzerland) coupled with a scanner, the maximum range of which was $100 \mathrm{~mm} \times$ $100 \mathrm{~mm} \times 5 \mathrm{~mm}$ in the $x-, y$-, and $z$-axes, respectively. The Rq measurements were performed by moving the atomic force microscopic probe (with a 4-6 mm height, a $48 \mathrm{~N} / \mathrm{m}$ spring constant, a $190 \mathrm{kHz}$ resonance frequency, a $25 \mu \mathrm{m} \times 25 \mu \mathrm{m}$ surface area, and a radius less than $10 \mathrm{~nm}$ ) across the buccal surface (middle $1 / 3$ ) of the tooth in tapping mode with a $10 \mathrm{~N}$ force. After determining the predebonding $\mathrm{Rq}$ with the atomic force microscope (three times per specimen), the data were calculated and averaged by using a commercially available software (C3000; Nanosurf, Liestal).

After confirmation of complete adhesive removal, all codes labeled on the experimental specimens were concealed with opaque plastic tape, and the 80 specimens were mingled into one large group. By using the atomic force microscope, their postdebonding Rq data were randomly evaluated.

\section{Scanning Electron Microscopy}

After incubation in a chamber (XPDB 701-54; Prodry, Shanghai, China) at $25^{\circ} \mathrm{C}$ and 40 to $60 \%$ humidity for 24 hours, each specimen was mounted on an aluminum stub and subjected to two coatings ( 60 min per round) of an $\sim 15 \mathrm{~nm}$ gold metallic sputter deposited layer (11425 AX; SPI-module Sputter Coater Module, Pennsylvania, United States).

A scanning electron microscope (S-3000N; Hitachi, Tokyo, Japan) at $5.0 \mathrm{kV}$ with a $10 \mathrm{~mm}$ working distance and a 500× magnification was used to evaluate the enamel characteristics. After obtaining the micrographs, three were randomly picked from each specimen. The characteristics were then graded by using a modified ESI described elsewhere, ${ }^{24}$ and the obtained numerical data were averaged.

\section{Statistical Analysis}

An assumption of normality was investigated by a Shapiro-Wilk test. All numerical data were subjected to statistical analysis by Statistical Package for the Social Sciences Statistics for Windows, version 23.0 (IBM; New York, United States). Descriptive statistics were used to determine the numerical data from the CRI and the modified ESI calculations. Differences among the polishing methods were compared with the one-way analysis of variance followed by Fisher's least significant difference test. The level of statistical significance was determined at $p<0.05$. 


\section{Results}

- Table 4 exhibits the CRI (mean and standard deviation) for each experimental group and the nonsignificant difference $(p=0.942)$ for each intergroup.

In the $2 \mathrm{D}$ and $3 \mathrm{D}$ atomic force microscopic images, the enamel surface in the control group was the smoothest (-Figs. 2A and 2B) among the samples herein, and those polished with Sof-Lex disc (-Figs. 2C and 2D) and sandblaster (-Figs. 2E and 2F) were slightly rough. The surface observed after processing with the tungsten carbide bur was moderately rough and possessed irregular hills (-Figs. 2G and $\mathbf{2 H}$ ). In addition to the severe roughness, some irregularly sharp peaks and deep grooves were visible in that polished with the white stone bur (-Figs. 2I and 2J).

- Table 5 displays the descriptive statistics for the preand postdebonding $\mathrm{Rq}$ from the atomic force microscopic measurements for each experimental group. A one-way analysis of variance among them disclosed no significant difference in the predebonding $(p=0.939)$, but there was a difference in the postdebonding $\mathrm{Rq}(p<0.05)$. The postdebonded enamel surfaces after polishing with the tungsten carbide and white stone burs were significantly rougher than those polished with the Sof-Lex disc and sandblaster $(p<0.05)$. A significant difference in the postdebonding $\mathrm{Rq}$ was not revealed $(p>0.05)$ between the Sof-Lex disc and sandblaster groups or between the tungsten

Table 4 Composite remnant index postdetachment of the bracket in each experimental group

\begin{tabular}{|l|l|}
\hline Group $(\boldsymbol{n})$ & Composite remnant index \\
\hline & Mean \pm SD \\
\hline Sof-Lex disc (20) & $3.70 \pm 0.80$ \\
\hline Sandblaster (20) & $3.70 \pm 0.73$ \\
\hline Tungsten carbide bur (20) & $3.80 \pm 0.62$ \\
\hline White stone bur (20) & $3.80 \pm 0.70$ \\
\hline p-Value & 0.942 \\
\hline
\end{tabular}

Abbreviation: SD, standard deviation. carbide and white stone bur groups. When compared with its predebonding $\mathrm{Rq}$, the postdebonding $\mathrm{Rq}$ was significantly increased $(p<0.001)$ in every experimental group.

Under a scanning electron microscope, a smooth surface without scratches was seen in the control group (-Fig. 3A). Homogeneous and smooth surfaces with some small shallow scratches were observed on the teeth polished with Sof-Lex disc (-Fig. 3B), while some mild, rough, and short scattered fine scratches with a few shallow pits were observed over the abraded area on the teeth polished with sandblaster (-Fig. 3C). Several consistent fine scratches were detected on the teeth polished with tungsten carbide bur (-Fig. 3D). Numerous fine scratches, some crack lines, and some obviously deep and coarse grooves parallel to the movement of the bur were visible on the teeth polished with white stone bur (-Fig. 3E).

Distributions and descriptive statistics of the modified ESI scores obtained from the 60 micrographs for each group are presented in - Table $\mathbf{6}$. Significant intergroup differences were observed in the scores $(p<0.05)$. The enamel surface in the control group significantly possessed the lowest score $(p<0.05)$ among the groups herein. Among all groups that underwent residual adhesive removal, the teeth polished with white stone bur significantly gained the highest score, followed by those polished with tungsten carbide bur, sandblaster, and Sof-Lex disc, respectively $(p<0.05)$. Significant differences in the means of modified ESI were detected among all paired groups ( $p<0.05$ ), but not between the sandblaster and Sof-Lex disc groups $(p>0.05)$.

\section{Discussion}

This is the first in vitro report of the enamel surface roughness following adhesive removal after orthodontic bracket detachment, particularly for teeth undergoing polishing by Sof-Lex disc and sandblaster, by using both atomic force microscopy and scanning electron microscopy. The quantitative and qualitative results have clearly shown significant differences in the enamel roughness among the different polishing methods.

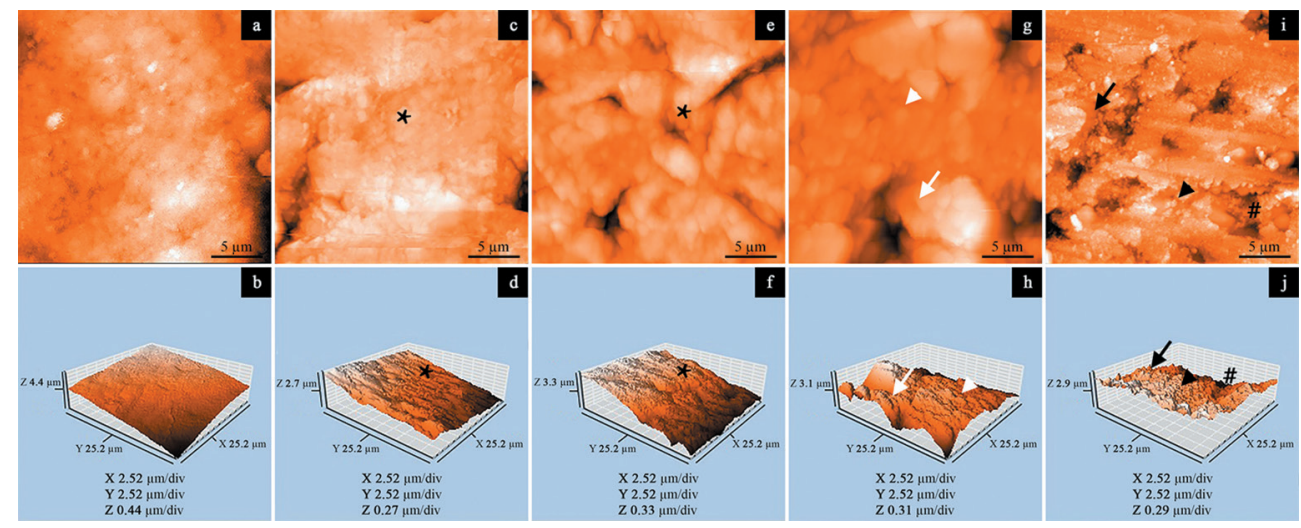

Fig. 2 Atomic force micrographs showing two-dimensional (upper row) and three-dimensional (lower row) post-thermocycled enamel surface in the control group (A, B) to be the smoothest, and those in the experimental groups to be slightly rough (asterisks) postpolishing by a Sof-Lex $\operatorname{disc}(\mathbf{C}, \mathbf{D})$ and a sandblaster (E, F), moderately rough (white arrowheads) with irregular hills (white arrows) by a tungsten carbide bur (G, H), and severely rough (dark arrowheads) with irregularly sharp peaks (dark arrows) and deep grooves (\#) by a white stone bur (I, J). 
Table 5 Pre and postdebonding root mean square (mean \pm standard deviation) in each experimental group

\begin{tabular}{|c|c|c|c|}
\hline \multirow[t]{2}{*}{ Group (n) } & \multicolumn{3}{|c|}{ Root mean square (nanometer) } \\
\hline & Predebonding & Postdebonding & $p$-Value ${ }^{*}$ \\
\hline Sof-Lex disc (20) & $47.70 \pm 14.92^{\mathrm{a}}$ & $51.65 \pm 13.67^{b}$ & $<0.001$ \\
\hline Sandblaster (20) & $46.08 \pm 19.08^{\mathrm{a}}$ & $53.86 \pm 19.09^{b}$ & $<0.001$ \\
\hline Tungsten carbide bur (20) & $47.35 \pm 17.41^{\mathrm{a}}$ & $63.36 \pm 12.24$ & $<0.001$ \\
\hline White stone bur (20) & $44.63 \pm 16.21^{\mathrm{a}}$ & $66.21 \pm 12.03^{c}$ & $<0.001$ \\
\hline$p$-Value ${ }^{* *}$ & 0.939 & $<0.05$ & \\
\hline
\end{tabular}

Note: Different uppercase letters indicate significant intracolumn differences by Fisher's least significant difference test at $p<0.05$ and intrarow differences by a paired $t$-test at $p<0.001$.

"Paired $t$-test.

"One-way analysis of variance.
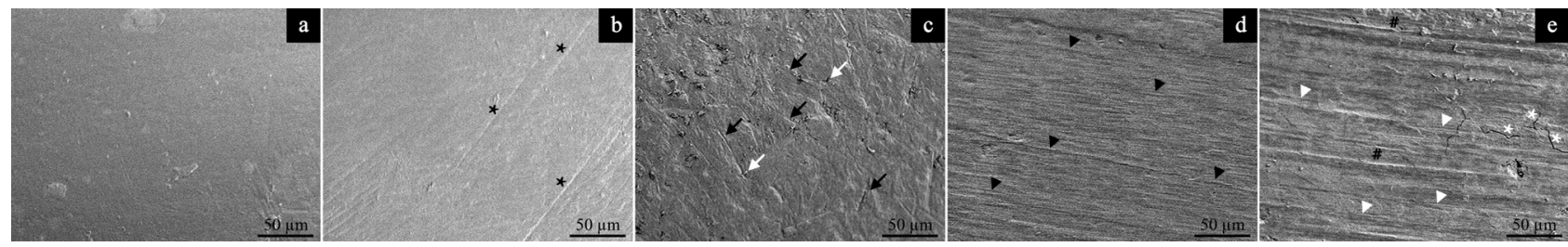

Fig. 3 Scanning electron micrographs showing post-thermocycled enamel surface without scratch in the control group (A) and those in the experimental groups (B-E) with (B) small shallow scratches (dark asterisks), (C) some mild, rough, and short scattered fine scratches (dark arrows) with a few shallow pits (white arrows), (D) several consistent fine scratches (dark arrowheads), and (E) numerous fine scratches (white arrowheads), some crack lines (white asterisks), and some obviously deep and coarse grooves (\#) parallel to the movement of the bur, postpolishing by a Sof-Lex disc, a sandblaster, a tungsten carbide bur, and a white stone bur, respectively.

Table 6 Modified enamel surface index (mean \pm standard deviation and score frequencies) in each group

\begin{tabular}{|l|l|l|l|l|l|}
\hline Group $(n)$ & \multicolumn{4}{|c|}{ Sodified enamel surface index } \\
\hline & Mean \pm standard deviation & \multicolumn{4}{|c|}{ Score frequencies (\%) } \\
\hline & & Score 0 & Score 1 & Score 2 & Score 3 \\
\hline Control (60) & $0.40 \pm 0.32^{\mathrm{a}}$ & $37(61.7)$ & $22(36.7)$ & $1(1.6)$ & $0(0)$ \\
\hline Sof-Lex disc (60) & $1.13 \pm 0.20^{\mathrm{b}}$ & $0(0)$ & $52(86.7)$ & $8(13.3)$ & $0(0)$ \\
\hline Sandblaster (60) & $1.17 \pm 0.20^{\mathrm{b}}$ & $0(0)$ & $50(83.3)$ & $10(16.7)$ & $0(0)$ \\
\hline Tungsten carbide bur (60) & $1.45 \pm 0.25^{\mathrm{c}}$ & $0(0)$ & $33(55.0)$ & $27(45.0)$ & $0(0)$ \\
\hline White stone bur (60) & $1.87 \pm 0.29^{\mathrm{d}}$ & $0(0)$ & $9(15.0)$ & $50(83.3)$ & $1(1.7)$ \\
\hline$p$-Value* & $<0.05$ & & & & \\
\hline
\end{tabular}

Note: Different uppercase letters indicate significant intracolumn differences by Fisher's least significant difference test at $p<0.05$.

* One-way analysis of variance.

Previous investigations into the enamel roughness postorthodontic polishing possess some restrictions. Despite its detailed images, scanning electron microscopy discloses subjective and nonquantifiable information. Unlike the electron microscopy, profilometry, rugosimetry, and atomic force microscopy enable topographical 3D examination of specimens and cause a minor amount of damage, if any, to the specimen surfaces. Profilometry ${ }^{8,9,17}$ and rugosimetry ${ }^{14}$ provide $3 \mathrm{D}$ information on the enamel roughness at the microscale, while atomic force microscopy provides 3D information at the nanoscale with high vertical and lateral resolutions. ${ }^{20}$ When compared with the methods in other documents, ${ }^{3,10}$ our utilization of both atomic force and scanning electron microscopes coupled with the determination of both the $\mathrm{Rq}$ and modified ESI, respectively, should provide additional reliable details on the postpolished enamel surfaces.
The predebonding Rq determined with the atomic force microscope did not possess a significant difference among the experimental groups, but the groups did have similarities in their initial enamel roughness. Because enamel can be destroyed during bracket detachment, bonding in orthodontics does not always require a very high bond strength. ${ }^{27}$ After thermocycling followed by bracket removal, the CRI did not possess a significant difference among the experimental groups and illustrated an equitable amount of residual adhesive on the tooth surfaces. Our relatively high CRI scores in all experimental groups indicated that $\sim 75 \%$ (or more) of the adhesive was left on the enamel surfaces ${ }^{12}$ and agreed with the results in a report. ${ }^{28}$ Consequently, enamel damage, if any, was minimized during our bracket detachment.

In each experimental group, the postdebonding Rq was significantly greater than the predebonding Rq. After the 
scanning electron microscopic observations, the modified ESI scores in all experimental groups were higher than those for the control group. These results were in accord with the documented data ${ }^{7,8,29}$ and showed that a greater postpolishing enamel roughness was created in all experimental groups, regardless of the methods used.

In the atomic force microscopic images and scanning electron micrographs, ascending grades of postdebonding enamel roughness were observed in Sof-Lex disc, sandblaster, tungsten carbide bur, and white stone bur, similar to the results from other investigations. ${ }^{9,12,30}$ When compared with a smooth surface in the control group from scanning electron microscopic analysis, a similar surface was observed for a tooth polished with a Sof-Lex disc, but with small scratches probably caused by aluminum oxide particles on the surface of the disc.

Short scratches and irregular pits on a relatively smooth surface after sandblasting were likely the results of the air pressure and aluminum oxide particles. The very rough surfaces obtained after polishing with the white stone and tungsten carbide burs herein were consistent with those reported earlier $^{12,30}$ and resulted from the rotational motion of the burs during polishing. However, polishing with a sandblaster and a tungsten carbide bur have been reported to cause no significant difference in postdebonding enamel roughness. ${ }^{17}$ Discrepancies between their results and ours might be due to differences in the instruments used (resolution) and the areas observed. Their results were obtained with profilometry (micrometer scale) and on the lower half of the buccal surface, while ours were obtained with atomic force microscopy and scanning electron microscopy (nanometer scale) and on the middle $1 / 3$ of the buccal surface. Postdebonding data obtained with the atomic force microscope showed significant differences in the enamel roughness among our four experimental groups, except for between the sandblaster and Sof-Lex disc groups and between the white stone bur and tungsten carbide bur groups.

With scanning electron microscopy, significant differences in the enamel roughness were observed among the five groups, except for between the sandblaster and Sof-Lex disc groups. The enamel roughness with a significant difference between the white stone bur and tungsten carbide bur groups was obtained as a result of the quantitative scanning electron microscopic analysis, but because there was no significant difference between them from the atomic force microscopic analysis, it was clear that there were some effects associated with the modified ESI scoring system and the different machines used in this research. Taken together, polishing with a sandblaster and a Sof-Lex disc for postorthodontic polishing purposes was favorably comparable, while polishing with a white stone and a tungsten carbide burs was questionably comparable.

Tooth surfaces with a large enamel roughness polarize adherence of bacterial plaque, ${ }^{31}$ leading to a drastically decreased $\mathrm{pH}$, chemical dissolution of enamel, and dental caries. A reduction in the roughness leads to a remarkable decrease in plaque formation. ${ }^{7}$ Consequently, an attempt to cause the lowest possible enamel roughness should be considered when an orthodontic treatment is conducted. An enamel surface roughness below $200 \mathrm{~nm}$ is necessary for the prevention of bacterial adhesion and plaque accumulation. ${ }^{31}$ Since the greatest range of enamel surface roughness created herein was with the white stone bur group and was $66.21 \pm 12.03 \mathrm{~nm}$ (mean \pm standard deviation), the four polishing methods seemed clinically acceptable for removing residual adhesives postdetachment of orthodontic brackets.

Limitations existed in this in vitro study. The studies that involve the convenience of orthodontist and preferences of patients for postorthodontic polishing methods are significant, and the time consumed for the complete removal of residual adhesives by each of them is essential for clinical deployment.

In addition, all biological responses were left unanswered in this study, particularly dentinal and pulpal reactions to conditions during and after the postorthodontic polishing methods. The conditions include applied force/pressure, induced temperature, and acidity/basicity level of the materials used. Clarifying such data are planned for future ex vivo and in vivo investigations.

\section{Conclusion}

Within the limitations of this study, no postpolished enamel surface was as smooth as its original morphology by any of the methods considered herein $(p=0.942)$. However, all methods resulted in a clinically acceptable enamel surface roughness, the greatest range of which was below $200 \mathrm{~nm}$. The sandblaster created as little enamel roughness as the Sof-Lex disc $(p>0.05)$, suggesting a possible replacement for each of them for postorthodontic polishing procedures.

\section{Funding}

This study was partially funded by the Faculty of Dentistry, Naresuan University, Phitsanulok, Thailand.

\section{Conflict of Interest}

None declared.

\section{References}

1 Proffit WR, Fields HW, Larson BE, Sarver DM. Contemporary Orthodontics. 6th edition. St. Louis: Mosby; 2019 321-355

2 Campbell PM. Enamel surfaces after orthodontic bracket debonding. Angle Orthod 1995;65(2):103-110

3 Janiszewska-Olszowska J, Szatkiewicz T, Tomkowski R, Tandecka K, Grocholewicz K. Effect of orthodontic debonding and adhesive removal on the enamel - current knowledge and future perspectives - a systematic review. Med Sci Monit 2014;20:1991-2001

4 Burapavong V, Marshall GW, Apfel DA, Perry HT. Enamel surface characteristics on removal of bonded orthodontic brackets. Am J Orthod 1978;74(2):176-187

5 Retief DH, Denys FR. Finishing of enamel surfaces after debonding of orthodontic attachments. Angle Orthod 1979;49(1):1-10

6 Zarrinnia K, Eid NM, Kehoe MJ. The effect of different debonding techniques on the enamel surface: an in vitro qualitative study. Am J Orthod Dentofacial Orthop 1995;108(3):284-293 
7 Karan S, Kircelli BH, Tasdelen B. Enamel surface roughness after debonding. Angle Orthod 2010;80(6):1081-1088

8 Ahrari F, Akbari M, Akbari J, Dabiri G. Enamel surface roughness after debonding of orthodontic brackets and various clean-up techniques. J Dent (Tehran) 2013;10(1):82-93

9 Mhatre AC, Tandur AP, Reddy SS, Karunakara BC, Baswaraj $\mathrm{H}$. Enamel surface evaluation after removal of orthodontic composite remnants by intraoral sandblasting technique and carbide bur technique: a three-dimensional surface profilometry and scanning electron microscopic study. J Int Oral Health 2015;7(Suppl 2):34-39

10 Shah P, Sharma P, Goje SK, Kanzariya N, Parikh M. Comparative evaluation of enamel surface roughness after debonding using four finishing and polishing systems for residual resin removal-an in vitro study. Prog Orthod 2019;20(1):18

11 Mahdavie NN, Manasse RJ, Viana G, Evans CA, Bedran-Russo AB. Enamel scarring by debonding burs: an SEM and profilometric study. J Clin Orthod 2014;48(1):14-21

12 Hong YH, Lew KK. Quantitative and qualitative assessment of enamel surface following five composite removal methods after bracket debonding. Eur J Orthod 1995;17(2):121-128

13 Webb BJ, Koch J, Hagan JL, Ballard RW, Armbruster PC. Enamel surface roughness of preferred debonding and polishing protocols. J Orthod 2016;43(1):39-46

14 Cardoso LA, Valdrighi HC, Vedovello Filho M, Correr AB. Effect of adhesive remnant removal on enamel topography after bracket debonding. Dental Press J Orthod 2014;19(6):105-112

15 Nishigawa G, Maruo Y, Irie M, et al. Various effects of sandblasting of dental restorative materials. PLoS One 2016;11(1):e0147077

16 Millett D, McCabe JF, Gordon PH. The role of sandblasting on the retention of metallic brackets applied with glass ionomer cement. Br J Orthod 1993;20(2):117-122

17 Kim SS, Park WK, Son WS, Ahn HS, Ro JH, Kim YD. Enamel surface evaluation after removal of orthodontic composite remnants by intraoral sandblasting: a 3-dimensional surface profilometry study. Am J Orthod Dentofacial Orthop 2007;132(1):71-76

18 Artun J, Bergland S. Clinical trials with crystal growth conditioning as an alternative to acid-etch enamel pretreatment. Am J Orthod 1984;85(4):333-340

19 Bishara SE, Trulove TS. Comparisons of different debonding techniques for ceramic brackets: an in vitro study. Part II. Findings and clinical implications. Am J Orthod Dentofacial Orthop 1990;98(3):263-273
20 Kubinek R, Zapletalova Z, Vujtek M, Novotný R, Kolarova H, Chmelickova H. Examination of dentin surface using AFM and SEM. Mod Res Educ Top Microsc 2007;2(15):593-598

21 Alessandri Bonetti G, Zanarini M, Incerti Parenti S, Lattuca M, Marchionni S, Gatto MR. Evaluation of enamel surfaces after bracket debonding: an in-vivo study with scanning electron microscopy. Am J Orthod Dentofacial Orthop 2011;140(5):696-702

22 Schiefelbein C, Rowland K. A comparative analysis of adhesive resin removal methods. Int $\mathrm{J}$ Orthod Milwaukee 2011;22(2):17-22

23 Pont HB, Özcan M, Bagis B, Ren Y. Loss of surface enamel after bracket debonding: an in-vivo and ex-vivo evaluation. Am J Orthod Dentofacial Orthop 2010;138(4):387.e1-387.e9

24 Oliver RG, Griffiths J. Different techniques of residual composite removal following debonding-time taken and surface enamel appearance. Br J Orthod 1992;19(2):131-137

25 Nanci A, Ten Cate's Oral Histology: Development, Structure, and Function. 9th ed. St. Louis: Elsevier; 2018 118-156

26 De Munck J, Van Landuyt K, Peumans M, et al. A critical review of the durability of adhesion to tooth tissue: methods and results. J Dent Res 2005;84(2):118-132

27 Khosravanifard B, Nemati-Anaraki S, Nili S, Rakhshan V. Assessing the effects of three resin removal methods and bracket sandblasting on shear bond strength of metallic orthodontic brackets and enamel surface. Orthod Waves 2011;70(1):27-38

28 González-Serrano C, Baena E, Fuentes MV, et al. Shear bond strength of a flash-free orthodontic adhesive system after thermal aging procedure. J Clin Exp Dent 2019;11(2):e154-e161

29 Mohebi S, Shafiee HA, Ameli N. Evaluation of enamel surface roughness after orthodontic bracket debonding with atomic force microscopy. Am J Orthod Dentofacial Orthop 2017;151(3):521-527

30 Osorio R, Toledano M, García-Godoy F. Enamel surface morphology after bracket debonding. ASDC J Dent Child 1998;65(5):313-317, 354

31 Bollen CM, Lambrechts P, Quirynen M. Comparison of surface roughness of oral hard materials to the threshold surface roughness for bacterial plaque retention: a review of the literature. Dent Mater 1997;13(4):258-269 